\title{
ACERCA DE LA SEMÁNTICA DEL COMPLETIVO/INCOMPLETIVO EN LAS LENGUAS MAYAS
}

\author{
IGOR VINOGRADOV \\ Instituto de Investigaciones Antropológicas, \\ Universidad Nacional Autónoma de México \\ happyjojik@gmail.com
}

The paper argues that completive and incompletive in Mayan languages are neither strictly aspectual nor strictly temporal categories; their meanings embrace several semantic domains. The term taxis is introduced. It is defined as a variety of tense that characterizes the event relative to some other event determined by the context without making reference to the moment of speech. It is proposed that the semantics of completive / incompletive is a combination of both taxis and aspectual meanings. Though the majority of Mayan languages apparently complies with this general semantic principle, there is significant variation within the family. This is, in the first place, the result of different internal structures of the verbal system and different sets of tense/aspect categories in particular languages.

Keywords: Mayan languages, aspect, tense, completive, incompletive

En este trabajo se argumenta que el completivo e incompletivo en las lenguas mayas no son categorías estrictamente aspectuales ni estrictamente temporales; 
sus significados abarcan varios dominios semánticos. Se introduce el término taxis que se define como una variedad de tiempo que caracteriza a un evento en relación a otro evento determinado por el contexto y sin hacer referencia al momento de habla. Se propone que la semántica del completivo/incompletivo consiste en la combinación de los significados de taxis y aspecto. Aunque la mayoría de las lenguas mayas parece seguir este principio semántico general, hay una variación muy significativa dentro de la familia que es, sobre todo, resultado de diferentes estructuras internas del sistema verbal y de la existencia de diferentes juegos de categorías de tiempo-aspecto en lenguas particulares.

Palabras clave: lenguas mayas, aspecto, tiempo, completivo, incompletivo

\section{INTRODUCCIÓN}

Las categorías del completivo e incompletivo son comunmente empleadas en la descripción de todas las lenguas modernas de la familia maya, aunque en algunos trabajos a veces se puedan encontrar otros términos para denominarlas. En la bibliografía sobre las lenguas mayas, aparecen amplias descripciones sobre la morfología y sintaxis de estas categorías, en las que se analizan los juegos de sus marcadores, la distribución de sus alomorfos y sus interconexiones con otras categorías. Pero la semántica de las marcas del completivo e incompletivo, por el momento, está insuficientemente descrita. No hay respuestas satisfactorias a preguntas muy simples a primera vista, tales como ¿qué significa el completivo?, ¿qué significa el incompletivo?, ¿cuál es la diferencia semántica entre el completivo e incompletivo? Lo único que no provoca duda es que las dos categorías forman parte esencial de la gramática verbal y del sistema gra- 
matical de "tiempo-aspecto", ${ }^{1}$ pero todavía no está claro si lo que expresan el completivo e incompletivo es tiempo, o aspecto, o ninguno de los dos, o tal vez ambos a la vez.

Lo que presento en este artículo es solo un primer acercamiento al problema. El estudio por el momento abarca pocas lenguas de la familia y no las analiza en toda la profundidad requerida para un estudio semántico de elementos gramaticales con significados tan generalizados y poco transparentes. Al escoger las lenguas de los ejemplos, intento incluir todas las subramas; sin embargo no se considera una muestra estadísticamente significativa. Aun así, con los resultados del trabajo se llega a conclusiones preliminares que pueden ser importantes tanto para futuros estudios de las lenguas particulares, como para investigaciones semánticas desde una perspectiva diacrónica o comparativa.

El presente estudio se basa en el análisis semántico de textos en lenguas mayas publicados en diferentes artículos y monografías, así como en colecciones de tradición oral. Algunos datos sobre la lengua q'eqchí fueron obtenidos mediante el trabajo con hablantes nativos.

El artículo consta de seis secciones. La parte introductoria $(\$ 1)$ incluye comentarios breves acerca de la evolución de la terminología empleada en relación con las categorías del completivo e incompletivo y de las definiciones de estas categorías que se proporcionan en diferentes gramáticas. Las propiedades morfológicas y sintácticas de las marcas del completivo/ incompletivo en varias lenguas mayas se presentan en $\$ 2$. En $\$ 3$ se argu-

${ }^{1}$ El término tradicional "tiempo-aspecto" no parece ser muy satisfactorio semánticamente, por las razones que voy a discutir a continuación. No obstante, a falta de alternativas mejores lo voy a utilizar en el presente trabajo. 
menta por qué el completivo y el incompletivo no se pueden considerar como categorías exclusivamente temporales, y tampoco como aspectuales. Se proponen diferentes soluciones al problema. Una de estas soluciones es emplear la noción de taxis que se discute en $\$ 4$. En $\$ 5$ se presenta un análisis comparativo que prueba las diferencias esenciales que se dan dentro de la familia lingüística entre la semántica del completivo y, en particular, el incompletivo. Las conclusiones se presentan en $\$ 6$.

\subsection{El problema terminológico}

Según las reconstrucciones históricas de Robertson (1992) y Houston et al. (2000), las categorías que actualmente se suelen llamar completivo e incompletivo existen en las lenguas mayas desde la proto-lengua. También existían en los tiempos coloniales cuando se escribieron las primeras gramáticas mayas. ${ }^{2}$ Los primeros autores describen "tiempos" en las lenguas mayas, del mismo modo que en las lenguas europeas. Lo mismo se encuentra en las primeras gramáticas científicas del siglo XIX y del inicio del siglo XX (véanse, por ejemplo, Stoll 1888 y Schuller 1925). Luego, se hizo evidente que las categorías verbales en las lenguas mayas difieren de sus "análogos" europeos, y no siempre expresan significados

2 Por el momento no está claro si el completivo e incompletivo se presentan en la lengua jeroglífica de inscripciones (maya clásico) o no. Kettunen \& Helmke (2005: 19) notan que entre los investigadores del maya clásico no hay una única opinión acerca de las categorías de tiempo ni aspecto; compárense diferentes puntos de vista en Houston (1997) y Law (2014). Tradicionalmente se opina que el sistema verbal del maya clásico se funda en distinciones aspectuales (Lacadena \& Davletshin 2013: 38). 
temporales. Al describir el sistema verbal maya, los autores más centrados en estudios comparativos empezaron a utilizar el término aspecto. $\mathrm{Al}$ parecer, el aspecto fue considerado un concepto lingüístico firmemente asociado con el tiempo; hasta entonces, no se había intentado analizar la semántica de las categorías verbales de manera más profunda. El hecho notorio de que en varias lenguas del mundo sí existen sistemas verbales evidentemente aspectuales sirvió como modelo para detectar sistemas de este tipo en las lenguas mayas también.

Actualmente, los términos más usuales para designar las categorías bajo consideración son completivo e incompletivo; también se usan en el presente artículo. No obstante, en la bibliografía a veces se reemplazan por diferentes términos, tales como pretérito y presente (Lehmann 1990), pasado y presente habitual (Stewart 1979), pasado y progresivo (England 1983), perfectivo e imperfectivo (Vázquez 2011). El último par de términos tiene mayor importancia porque es de uso mucho más general en la lingüística, $\mathrm{y}$, además, tipológicamente parecen ser los más similares a los del completivolincompletivo en las lenguas mayas. Fuera de la tradición mayista, los términos completivo e incompletivo no son muy comunes y suelen utilizarse con significados muy particulares (véase la discusión en Watatani 1995: 16-18). Por ejemplo, Bybee et al. (1994: 54) usan el término completivo para denominar uno de los significados aspectuales elementales, a saber, "hacer algo completamente y hasta su terminación”, y esta concepción es mucho más reducida que la que se suele entender utilizando el término perfectivo. Welmers (1974: 345), al describir la lengua africana igbo, se refiere con incompletivo a una marca que combina los significados de acción habitual y de una acción que se está continuando independientemente del tiempo. 
Varios autores mencionan explícitamente la coincidencia terminológica que se da en varias lenguas mayas: "Los términos completivo e incompletivo son aproximadamente equivalentes al perfectivo e imperfectivo" (England 2009: 213). Sin embargo, igualar el completivo/incompletivo en las lenguas mayas con las categorías más reconocidas tipológicamente del perfectivo/imperfectivo no parece ser la mejor solución. Primero, porque tampoco hay uniformidad en lo que concierne a los significados del perfectivo/imperfectivo en las lenguas del mundo: los usos del perfectivolimperfectivo "difieren aún entre las lenguas relacionadas genéticamente, y las diferencias están lejos de ser triviales" (Gvozdanović 2011: 785); segundo, porque, como mostraré a continuación, estas categorías en las lenguas mayas no son exclusivamente aspectuales, lo cual las distingue del estándar tipológico para el perfectivo/imperfectivo.

\subsection{Definiciones posibles}

Las descripciones gramaticales de diferentes lenguas mayas definen las categorías del completivo e incompletivo de una manera superficial. Comúnmente, se menciona el concepto de "terminación” de una acción; por ejemplo, en acateco "el completivo es el aspecto que hace referencia a las acciones que ya se terminaron, ya sea de forma inmediata o en un período de tiempo mayor", mientras que "el incompletivo es el aspecto que hace referencia a las acciones que no están terminadas" (Zavala 1992: 69-70). Para otras lenguas de la familia se proporcionan definiciones muy similares: en chol el completivo "indica que las acciones denotadas por predicados verbales se han terminado” (Vázquez 2011: 194); 
en tzeltal, "el aspecto completivo sirve para expresar eventos considerados como un todo acabado o completo" y "el aspecto incompletivo presenta los eventos como no terminados, es decir, en curso de realización o de vigencia, o como habituales" (Polian 2013: 154-160). Es importante notar que la noción de terminación por sí misma se puede referir tanto al aspecto verbal como también al tiempo, según la ubicación relativa del punto de referencia, o, en otras palabras, según el marco en que se observa la situación.

Hay una tendencia a definir el incompletivo con base en una lista, a veces bastante larga, de significados elementales. Por ejemplo, Craig (1977: 60-63) indica seis usos elementales del incompletivo en el jacalteco (poptî): 1) una acción que ocurre en el presente; 2) tiempo presente de narración; 3) presente habitual; 4) "presente pasado" para acciones que empezaron en el pasado pero todavía están ocurriendo; 5) expresiones de simultaneidad; y 6) construcciones futuras. Una lista, aunque menos extensa, también aparece en Dayley (1985: 80) para el incompletivo en tz’utujil: 1) aspecto habitual; 2) tiempo futuro inmediato; 3) pasado narrativo en discurso. Es importante señalar que para el completivo no se encuentran listas de significados elementales como estos. Este hecho puede servir como evidencia de la complejidad semántica del incompletivo, a diferencia del completivo.

\section{RASGOS MORFOLÓGICOS Y ESTRUCTURALES}

Antes de empezar a analizar la semántica del completivo y el incompletivo, hay que hacer un breve comentario sobre la morfología de estas cate- 
gorías en diferentes lenguas de la familia, así como sobre la estructura del sistema gramatical de tiempo-aspecto en general. Aunque el completivo/incompletivo se presentan en todas las lenguas de la familia, como fue notado en $\$ 1$, su marcación morfológica varía considerablemente. $\mathrm{Ni}$ para el completivo ni para el incompletivo se reconstruye una protomarca común de la cual se deriven todas las marcas modernas en diferentes lenguas mayas. Al contrario, las marcas que existen hoy en día son evidencias de varios procesos de gramaticalización.

En general, las marcas ocupan la posición inicial en el complejo verbal, y son elementos predicativos, partículas prepositivas, proclíticos o prefijos según la lengua particular. Solo dos lenguas mayas -huasteco y chontalexpresan distinciones gramaticales de tiempo-aspecto por medio de sufijos. Las lenguas de la rama yucatecana y chol tienen un sistema de doble marcación, en el que una categoría se marca por un sufijo y por un elemento prepositivo al mismo tiempo. En (1-3) se muestra el contraste entre las tres diferentes opciones para la estructura morfológica de esta categoría:

(i) Sipakapense

k-in-kom

INC-ISG.ABS-morir

'moriré' (Barrett 1999: 313, ej. 37)

(2) Huasteco

in utx-a-al

3ERG decir-TR-INC

'decía' (Kondic 2012: 52, ej. 9) 
(3) Mopán

walak u=su't-ik u-bahil

INC $3 \mathrm{~A}=$ cambiar-INC 3POSS-REFL

'se convierte' (Ulrich \& Ulrich 1986: 33)

La única lengua que no tiene marcas de completivo ni de incompletivo es el chortí. No obstante, ello no significa que estas categorías no existen en chortî́. La dicotomía completivo/incompletivo es relevante en chortí solamente para verbos intransitivos y en el nivel morfosintáctico se manifiesta por el afijo pronominal, es decir, se expresa cumulativamente junto con persona y número del sujeto. En chortî hay dos paradigmas de marcadores pronominales absolutivos: el "juego B” se usa para expresar el completivo mientras que el “juego C” está reservado para los usos incompletivos. Compárese (4a-b).

(4) Chortí'
a. sajmi ixin-en
$\mathrm{t}=$ uy-otot
in-k’ajti
hoy ir-1sG.B.COM
PREP $=3$ POSS-casa ISG.A-pedir
'Hoy fui a su casa a pedir [dinero]' (Pérez 1996: 27)
b. ejk'ar i< $>$ xin ta chonma'r mañana ir $<1$ sG.C.INC $>$ PREP mercado 'Mañana iré al mercado' (Pérez 1996: 19)

Las categorías del completivo e incompletivo forman parte del sistema verbal de tiempo-aspecto. Estos sistemas también difieren mucho en 
las lenguas mayas, y esa diferencia no solo involucra rasgos morfológicos sino también el juego de categorías y de las marcas que las expresan. Hay lenguas con sistemas binarios de tiempo-aspecto, como las lenguas tzeltalanas, chontal y chortí. En estas lenguas el sistema de tiempo-aspecto se representa por la oposición entre el completivo e incompletivo. Pero en la mayoría de las lenguas de la familia el sistema no se limita por dichas categorías. En huasteco, por ejemplo, el perfecto juega el papel del tercer miembro del paradigma de tiempo-aspecto (Watatani 1995: 47); en las lenguas q’anjob'alanas el lugar del tercer miembro lo ocupa el futuro o "potencial" (Zavala 1992: 71; Buenrostro 2013a: 119). Los paradigmas en las lenguas de la rama k'icheana normalmente incluyen el optativo y prohibitivo ("optativo negativo"), pero también pueden incluir el futuro (o "potencial") y una categoría adicional del completivo que suelen llamar "completivo remoto". En q'eqchí" -que representa el caso extremo dentro de la rama k'icheana- el paradigma de tiempo-aspecto consta de seis miembros (Stewart 1979).

Los sistemas de doble marcación morfológica se distinguen no solo por la estructura formal, sino también por el juego de categorías. Por ejemplo, en chol hay una categoría que Vázquez (2011: 200) llama "irrealis in the preterite". Esta indica una acción en el pasado que estaba a punto de suceder pero por uno u otro motivo no sucedió. Para designar este significado Kuteva (1998) propuso el término "avertivo". El sistema de tiempo-aspecto en las lenguas yucatecanas es mucho más amplio que el de chol. Sin entrar en detalles, solo menciono que entre los autores no hay unanimidad acerca de la cantidad de categorías que forman el sistema de tiempo-aspecto-modo en las lenguas de la rama 
yucatecana. ${ }^{3}$ Analizando el caso del maya yucateco, por ejemplo, Bohnemeyer (1998: 25) presenta una lista de 15 categorías, Briceño (2006: 30) enumera 17 marcas, mientras que Hofling (2006: 389-391) cita 19 marcadores. Entre estas categorías hay algunas que denotan varios matices modales, irreales o de distancia temporal.

\section{LA SEMÁNTICA: ¿'TIEMPO O ASPECTO?}

En esta sección voy a presentar los argumentos a favor de no considerar el completivo e incompletivo como categorías aspectuales $(\$ 3.1)$ y a favor de no considerarlos tampoco como categorías temporales (\$3.2). Las posibles interpretaciones alternativas se enumeran en $\$ 3.3$.

\subsection{Interpretación aspectual}

Los usos del completivo e incompletivo en las lenguas mayas tienen peculiaridades muy importantes. El completivo casi no se usa en relación al tiempo futuro, mientras que el incompletivo casi no se usa en relación al tiempo pasado. Un evento futuro, aunque sea un evento puntual desde el punto de vista "aspectológico", normalmente no puede llevar marca del completivo. Hasta donde sé, esto es cierto con algunas restricciones para todas las lenguas y se menciona en varias gramáticas; por ejemplo, Polian (2013: 156) nota que en tzeltal "el completivo en oraciones

3 Al hablar de sistemas verbales en las lenguas yucatecanas es necesario incluir también la noción de modo ya que varios significados modales se expresan dentro del mismo paradigma junto con significados temporales y aspectuales. 
independientes suele ser incompatible con una referencia explícitamente futura". Según la lengua particular y el juego de marcas de tiempo-aspecto del que esa lengua dispone (véase $\$ 2$ ), una acción puntual futura se indica con el incompletivo (5), con el potencial (6) o con alguna categoría modal (7).

(5) Chontal

ida kä=k'ux-e'-et

aquí ISG.A=comer-INC-2SG.B

'Aquí te comeré' (Knowles \& Hernández 1989: 227)

(6) Мам

ok $\quad k^{\prime}$-eel $=\mathrm{a} \quad \mathrm{t}$-u'n $\quad \mathrm{n}$-maan $=\mathrm{a}$,

POT matar-POT=2SG 3SG.POss-por ISG.POss-papá= ISG

'Te va a matar mi papá (England \& Ortiz 1985: 18, ej. 199)

(7) Yucateco

eyaj xtúuchaj, je’ u=bis-ik-ech kisin=e'

eh mono ASEG 3A=llevar-INC-2SG.B diablo=ENCL

'Oye mono, te llevará el diablo' (Andrade \& Máas Collí 1999: 331)

El uso del completivo en (5)-(7) modificaría la lectura de estas oraciones; los eventos se entenderían como ya ocurridos.

Un evento durativo o habitual -es decir, un evento que, aspectualmente, se espera encontrar marcado por el incompletivo- normalmente lleva la marca de completivo cuando se refiere al pasado (8)-(9). 
(8) TzotzIL
te i-levan-at
li ants=e
s-junul
ak'ubal
allí COM-abusar-PASs
DEF mujer=ENCL 3SG.POSs-todo
noche 'La mujer fue abusada toda la noche' (Laughlin 1977: 76)

(9) Tzeltal

$\begin{array}{llllll}\text { ay } \quad \text { yan-tik } & \text { la } & \text { s-maj-ik } & \text { sok } & \text { la } \\ \text { EXIST otro-PL } & \text { COM } & \text { 3SG.ERG-golpear-PL } & \text { y } & \text { COM } \\ \text { X-chik'-ik } & & & & \end{array}$

3SG.ERG-quemar-PL

'Otros lo golpeaban y lo quemaban' (Relatos tzeltales 2002: 18)

Estas dos peculiaridades bien conocidas pero subestimadas no permiten analizar el completivo/incompletivo en términos meramente aspectuales. El aspecto verbal por definición no depende del tiempo, ya que describe nada más la estructura interna de una acción sin hacer ninguna referencia temporal. Es decir, si las categorías del completivo e incompletivo en las lenguas mayas fueran exclusivamente aspectuales, no habría restricciones temporales que limiten sus usos.

\subsection{Interpretación temporal}

Dado que el completivo y el incompletivo están restringidos temporalmente, entonces ¿por qué no pueden considerarse categorías temporales? El problema es que dos de los rasgos mencionados arriba no son reglas estrictas, ya que hay algunos contextos muy especiales en los que pueden 
ser violadas: cuando el completivo se usa en relación al tiempo futuro o presente y cuando el incompletivo se usa en relación al tiempo pasado. Un caso común en el que un verbo que no se refiere al pasado aparece marcado por el completivo se atestigua en las cláusulas subordinadas de condición real (10-11).

(10) Tzotzil

ti_mi i-kuch k-u'un=e, ta $\mathrm{x}$-bat

si COM-acarrear 1sG.POss-causa=ENCL INC INC-irse

k-al-be li a-tot

1SG.ERG-hablar-APPL DEF 2POss-padre

'Si gano [lit. "acarrea mi causa"], voy a hablarle a tu padre' (Laughlin 1977: 34)

(11) CHOL

mi tsi' k'el-e-yob ti mal mukonibäl iy-alobil, si COM ver-COM-PL PREP adentro sepulcro 3POSs-hijo mi' chok-ob ochel lum

INC tirar.INC-PL DIR lodo

'Si sus hijos miran adentro del sepulcro, tiran lodo adentro' (Whittaker \& Warkentin 1965: 117)

Aparte de las cláusulas condicionales, existen otros contextos que comparten este comportamiento. Por ejemplo, Polian (2013: 155) menciona que el empleo del completivo en tzeltal "subraya que el evento [futuro] es algo ya decidido, ineluctable, casi como si ya hubiera ocurri- 
do". Además, el completivo se usa en las fórmulas de despedida y también "para destacar una secuencia de eventos", cuando el completivo "permite resaltar el hecho de que los eventos no se empalman y que forman una secuencia: termina uno, empieza otro" (Polian 2013: 155).

Refiriéndose a eventos pasados, las marcas del incompletivo pueden aparecer en discurso narrativo. Dentro de la familia maya, hay lenguas que emplean el incompletivo en narraciones más frecuentemente-como mam o uspanteko- y otras que lo emplean menos frecuentemente como tzotzil o sakapulteko- (Vinogradov 2014a: 51-52). No obstante, los empleos del incompletivo para marcar un evento aspectualmente perfectivo que pertenece al pasado se encuentran en narraciones con mayor o menor frecuencia por todas partes (12-14).

(12) MAм

tzinxax $\quad \mathbf{n}=$ jaa=tz t-jak'u-'n $\quad$ xjaal aj

luego INC=DIR=DIR 3SG.ERG-jalar-TR persona DEM t-k'uxb'il

3SG.POSs-cuchillo

'Luego la persona sacó su cuchillo' (England \& Ortiz 1985: 5, ej. 43)

(13) $\mathrm{CHOL}$

$\mathbf{m u}=\mathrm{x} \quad \mathrm{i}-\mathrm{kaj} \quad \mathrm{i}$-pejk-an

INC=ya 3A-empezar 3A-hablar-INC

'Entonces empezó a hablar[le]' (Whittaker \& Warkentin 1965: 125) 
(14) Tzotzil

ch-k'ot la ta mukenal

INC-llegar QUOT PREP cementerio

'Dicen que llegó al cementerio' (Laughlin 1977: 28)

Hay otros casos en los que el incompletivo es compatible con referencia al pasado. Por ejemplo, en tzotzil hay partículas especiales que autorizan el empleo del incompletivo en un contexto temporalmente impropio (Vinogradov 2014a: 44).

\subsection{Alternativas posibles}

Es evidente pues, que el completivo y el incompletivo en las lenguas mayas no son categorías puramente aspectuales, ni tampoco son categorías puramente temporales. Entonces, ¿cómo se puede analizar su semántica? En general, hay dos opciones. Primero, se les puede considerar como aspectuales en unas circunstancias y como temporales en otras. Aceptando esta opción, hay que hacer una suposición de que existen algunas condiciones que determinan en cada contexto particular si el aspecto supera al tiempo o, por el contrario, el tiempo supera el aspecto. Segundo, se puede aceptar que no son aspectuales ni temporales, y entonces hay que entender cuál es el ámbito semántico cuyos significados realmente se expresan por estas categorías. La segunda alternativa implica al menos dos soluciones posibles: se puede analizar la semántica del completivo/incompletivo en términos modales o se puede emplear la noción de tiempo relativo o taxis. 
De hecho, la primera hipótesis no es tan innovadora porque la interconexión entre aspecto y tiempo se registra tipológicamente en varias lenguas del mundo (Dahl 1985: 79-83). Un ejemplo muy común es cuando el perfectivo aparece restringido por el tiempo pasado y resulta una categoría a la que se suele llamar aoristo (consúltense Comrie 1976: 71-73 y Dahl 1985: 80 para más detalles). No obstante, los rasgos semánticos del completivo maya no corresponden completamente con los del aoristo; como fue mostrado en $\$ 3.2$ (ejemplos 10-11), el completivo en las lenguas mayas no necesariamente se emplea con referencia al tiempo pasado.

Otra posibilidad es interpretar el completivo/incompletivo como una distinción que se basa en propiedades modales y cuya semántica en realidad no tiene nada que ver con aspecto ni tiempo. DeChicchis (1996: 69) propone una interpretación modal del sistema de tiempo-aspecto en q'eqchî", sugiriendo que "la mayoría de los hablantes sigue distinguiendo predicados según asertividad, predictibilidad y optatividad". El sistema verbal en q'eqchî' es muy amplio en comparación con otras lenguas de la familia $(\$ 2)$, y la presencia de categorías evidentemente modales (potencial, optativo) puede favorecer considerablemente la interpretación modal. Para otras lenguas, no se han propuesto hipótesis parecidas, que yo sepa.

Para las lenguas con distinción binaria entre el completivo e incompletivo, uno puede sugerir tratar estas categorías como la dicotomía realis/ irrealis que es común para las lenguas de América del Sur (Müller 2013: 135-141). ${ }^{4}$ La referencia al futuro que en las lenguas mayas comúnmente

4 Roberts (1990), Givón (1994) y otros autores consideran al irrealis como una categoría modal. No obstante, según las evidencias obtenidas mediante estudios tipológicos, el ámbito de irrealis en las lenguas del mundo es mucho más amplio que el dominio de modalidad, y por 
se expresa por el incompletivo sin tomar en cuenta los rasgos aspectuales de la acción, puede considerarse desde el punto de vista modal; véase Palmer (2001: 104-106). El incompletivo maya podría pues ser identificado con la modalidad irrealis: "[los] eventos futuros son frecuentemente clasificados como irrealis [...] ya que no han ocurrido (todavía)" (Mithun 1995: 378). Esta propuesta se apoya también en otros estudios tipológicos; por ejemplo, Elliott (2000: 70) menciona, entre los objetivos frecuentes de marcación con irrealis, en primer lugar, a los eventos potenciales. Es notable que en segundo lugar se mencionan eventos condicionales que en las lenguas mayas también permiten el empleo del incompletivo. Si el incompletivo parece ser buen "candidato" para el irrealis, el completivo - siendo el otro miembro de la oposición- será el realis. Sin embargo, la dicotomía del completivo/incompletivo tiene una correspondencia con la del realis/irrealis en la mayoría de los contextos, pero no en todos. El mayor problema aparece con el significado progresivo, es decir, cuando una acción se está desarrollando en el presente. Este significado es considerado por Elliott (2000: 68) el empleo más básico de la categoría de realis: "un evento que se percibe como sucediendo o al menos como iniciado, prototípicamente se marcará por realis". Pero en muchas lenguas mayas los eventos que están en progreso no llevan marca del completivo; al contrario, pueden ser marcados por el incompletivo (15-16) o por una marca especial del progresivo si este se representa como una categoría separada en la lengua particular (17).

lo tanto la distinción realis/irrealis no necesariamente debe ser considerada como un subtipo de distinción modal (Plungian 2004: 16-17). 
(15) IXIL

ni kuxh un-chuk un-b'ooj w-aq'on INC solo ISG.A-buscar ISG.POSS-poco ISG.POss-trabajo 'Solo estoy buscando un poco de trabajo.' (Ayres et al. 2001: 272)

(16) Chontal

$\mathrm{u}=$ yer-o mundo

3A=caer-INC mundo

'El mundo está cayendo.' (Knowles \& Hernández 1989: 232)

(17) Chus

lan honh-s-mil-an-cham heb' winh ejersito PROG IPL.ABS-3ERG-matar-SUF-morir 3PL CLF:hombre ejército 'Nos está matando el ejército.' (Buenrostro 2013b: 41, ej. 44)

Así pues, el completivo no aparece en el contexto prototípico realis. La última opción es considerar el completivo/incompletivo maya como categorías que expresan significados del dominio semántico de tiempo relativo o taxis. Jakobson (1957: 135) fue el primer autor que introdujo el término taxis que "caracteriza el evento narrado en relación a otro evento narrado sin referencia al evento del habla". Este término no fue admitido de modo general y "no ha jugado papel importante en la discusión sobre tiempo y aspecto" (Zeisler 2004: 46). A veces se emplea en descripciones de sistemas verbales en las lenguas eslavas, o para explicar las reglas de la coordinación de tiempos en las lenguas indoeuropeas (por ejemplo, consúltese Martínez 1995 para el caso del griego antiguo). En el mismo sentido, a veces se emplea el término tiempo 
relativo que revela adecuadamente la diferencia con el tiempo absoluto: Comrie (1985: 56) traza el límite entre tiempo relativo y tiempo absoluto de acuerdo con la disposición del punto de referencia que establece el marco para determinar la ubicación de la situación en la escala temporal. Para el tiempo absoluto es el momento del habla, mientras que para la taxis es un momento determinado por el contexto que no coincide necesariamente con el momento del habla. Los términos taxis y tiempo relativo parecen ser sinónimos. En lo sucesivo se emplea el término taxis para evitar confusión entre tiempo absoluto y tiempo relativo. Además, el término tiempo relativo parece ser confuso por sí mismo ya que la concepción del tiempo de todas formas presupone algo de relatividad.

\section{El DOMINIO SEMÁNTICO DE TAXIS}

En $\$ 3.1$ y $\$ 3.2$ se mostró por qué no se puede describir la semántica del completivo/incompletivo en las lenguas mayas en términos aspectuales ni temporales. En $\$ 3.3$ se plantearon las hipótesis acerca de cómo se podría analizar estas categorías. De las cuatro hipótesis mencionadas arriba la más acertada parece ser la última, que se basa en la noción de taxis.

\subsection{Los significados de taxis: anterioridad, simultaneidad y posterioridad}

Dado que el completivo y el incompletivo se restringen temporalmente, el problema con la interpretación temporal del completivo/incompletivo aparece cuando uno se enfrenta con las excepciones de las reglas que predeterminan el empleo del incompletivo en relación al presente o 
futuro, y el empleo del completivo en relación al pasado. Efectivamente, la noción de taxis explica muy bien los casos excepcionales en los que el completivo sí se combina con la referencia temporal del presente/futuro y el incompletivo se combina con la referencia temporal del pasado; pero, el punto de referencia no debe ser el momento de habla como en el caso de tiempo absoluto, sino otro momento particular dado por el contexto. Este otro momento puede coincidir con el momento del habla o no. Voy a mostrar esta diferencia con el ejemplo de la oración condicional (10) que se repite a continuación.

(10) Tzotzil

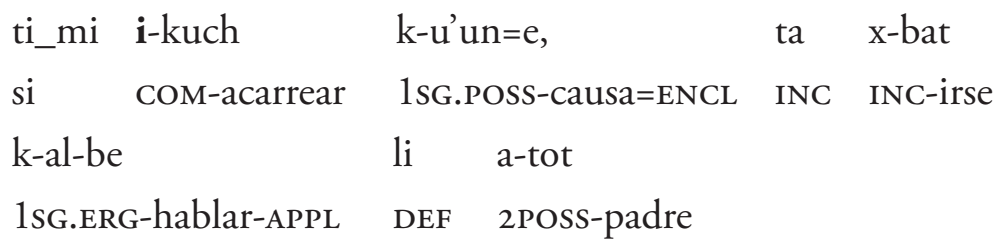

'Si gano [lit. "acarrea mi causa"], voy a hablarle a tu padre' (Laughlin 1977: 34)

Hablando de tiempo absoluto, la oración (10) pertenece al futuro, ya que ambos eventos al momento de hablar todavía no han sucedido y ni siquiera empezaron a suceder. Sin embargo, el primer evento (ganar) evidentemente precede al otro (hablar) por lo que hablando de taxis, lo que expresa la primera forma verbal marcada por el completivo es anterioridad en relación al otro evento mencionado en la cláusula principal. La relación temporal con el momento del habla en este caso no importa. Así pues, el completivo aquí tiene el significado de anterioridad, mientras 
que el incompletivo expresa simultaneidad o posterioridad; estos tres significados pertenecen al dominio semántico de taxis. De hecho, parece que precisamente la distinción entre anterioridad, por un lado, y simultaneidad o posterioridad, por otro lado, es lo que se sobreentiende bajo la noción de terminación que muy comúnmente se usa en diferentes gramáticas mayas cuando se trata del completivo/incompletivo (véase $\$ 1.2$.).

Normalmente, el punto de referencia temporal que es crucial para determinar el significado de la taxis coincide con el momento del habla, y en este caso no hay diferencia entre tiempo absoluto y taxis. Pero el punto de referencia puede ser trasladado hacia el futuro o, más comúnmente, hacia el pasado, como en el caso de subordinación condicional, y así se explican otras excepciones mencionadas en $\$ 4.2$. El empleo del incompletivo en discurso narrativo se explica por el traslado del punto de referencia desde el momento en que se habla hacia el "tiempo narrativo" (el momento en que suceden los eventos narrados). Este traslado no lleva ninguna marca específica y se determina pragmáticamente por la situación de narración. Así pues, un evento narrado es simultáneo al punto referencia y por eso permite marcación incompletiva aunque sea anterior al momento del habla. En Vinogradov (2014a: 50) se hace notar que este fenómeno en las lenguas mayas es hasta cierto grado similar al así llamado "presente histórico" en las lenguas europeas. El empleo del completivo para marcar una secuencia de eventos en presente o futuro mencionado por Polian (2013: 155) no encuentra buena explicación si el completivo/incompletivo fueran categorías aspectuales, pero se explica muy bien tomando en cuenta la noción de taxis. Lo que es indicado por el completivo es la anterioridad de un evento en la secuencia en referencia a otro evento de la misma 
secuencia; el empleo del completivo en este caso subraya que "los eventos no se empalman [...]: termina uno, empieza otro".

\subsection{Combinación semántica de taxis y aspecto}

A pesar del razonamiento presentado en $\$ 4.1$, analizar los sistemas verbales mayas apoyándose solo en la noción de la taxis sería una simplificación injustificada. La razón de ello es una variación en la marcación: los casos descritos en $\$ 3.2$ como excepciones de las reglas básicas de la incompatibilidad del completivo e incompletivo con referencia al futuro y al pasado respectivamente casi siempre tienen alternativas no excepcionales, esto es, cuando el completivo y el incompletivo son posibles en un mismo contexto. Esto no solo concierne a las narraciones en las que los marcadores gramaticales desempeñan funciones discursivas y se pueden desviar de sus significados primarios, sino también a otros contextos. Por ejemplo, las oraciones subordinadas de condición real presentadas en (1011) no necesariamente deben ser marcadas por el completivo; también se puede emplear el incompletivo sin visibles cambios semánticos (18-19).

(18) K'ICHÉ'

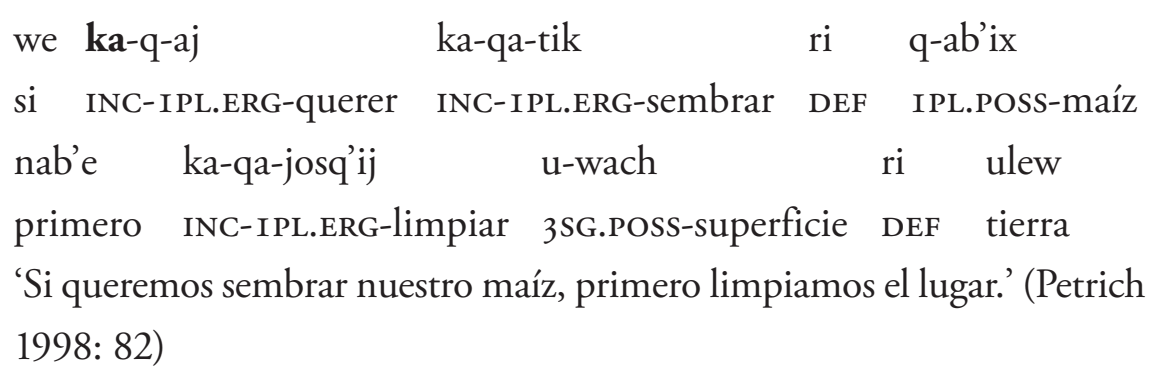


(19) TzotziL

ti_mi ch-a-k'an=e che'e, ch-a-j-timan

si INC-2ERG-desear=ENCL entonces INC-2ABS-IERG-atar

komel li' ta $y$-ok te'=e

DIR:quedarse aquí PREP 3POSS-pie árbol=ENCL

'Entonces, si quieres, te dejo atado aquí debajo del árbol.' (Laughlin 1977: 66)

Los "contraejemplos" (18-19) son buenos argumentos a favor de no considerar el completivo/incompletivo como categorías exclusivamente de la taxis. Otro contexto en que el completivo parece ser intercambiable con el incompletivo sin afectar los rasgos semánticos es un evento habitual en el pasado que ya no es actual en el presente, es decir, un evento habitual que fue sustituido por otro evento; compárese (9) y (20).

(20) Tzeltal

a. ta jujun sab ya x-bajt ta a'tel PREP cada mañana INC INC-irse PREP trabajo 'Cada mañana se iba al trabajo' (Pérez et al. 1994: 289)

b. ya y-al-ik ta namey te ya $x$-k'opoj INC 3ERG-decir-PL PREP antes REL INC INC-hablar te ts'i'=e

DEF perro $=$ ENCL

'Dicen que el perro antiguamente hablaba' (Alarcón et al. 1997: 227) 
Este rasgo no es característico solo del tzeltal. Lo mismo sucede, por ejemplo, en chol (21).

(21) $\mathrm{CHOL}$

a. tyi i-pejtyälel k'iñ che' mi' i-k'oty-el tyi PREP 3POSS-todo día CONJ INC 3A-llegar-INC PREP iy-otyoty=i

3POSS-Casa $=$ ENCL

'Siempre que regresaba a su casa [...]' (Relatos choles 2002: 46, 48)

b. kabäl kixtyañuj-ob tyi lak-lumal mi' mucho persona-PL PREP IPL.INCL.POSS-pueblo INC i-k’uxb-iñ-oń 3A-amar-INC-IB

'Muchas personas del pueblo me querían' (Relatos choles 2002: 42)

La interpretación del completivo/incompletivo como taxis implica el criterio de ubicación del punto de referencia en la escala temporal que por sí mismo es muy vago. En la mayoría de los casos la lengua no dispone de ninguna marca especial para indicar este punto. Por consecuencia, casi nunca se puede confirmar con exactitud que el momento de referencia coincide con uno u otro evento. Pero aun así, los ejemplos de ausencia de contraposición entre el completivo y el incompletivo (18-21) hacen claro que no siempre es correcto analizar el completivo como una categoría de taxis que expresa anterioridad ni el incompletivo como una categoría de taxis que expresa simultaneidad o posterioridad. Se 
supone que, aparte de los significados del dominio semántico de taxis, dichas categorías expresan significados aspectuales. En algunos contextos prevalece la semántica de taxis y en los otros la de aspecto. Pero cuáles son los contextos del primer tipo y cuáles son los del segundo, no está claro.

Las categorías que abarcan dominios de tiempo y de taxis a la vez son muy bien conocidas en lenguas europeas. Un ejemplo posible se presenta en el así llamado "futuro en el pasado" que manifiesta el conjunto del significado temporal de pasado y el significado de posterioridad que pertenece al dominio de taxis. En español, por ejemplo, así es el significado gramatical de la forma irías en la oración Ayer me dijiste que irías al mercado que, aparte de expresar la información sobre persona, número y modo, indica cumulativamente el tiempo pasado y la taxis de posterioridad. Con respecto a la taxis, irías se opone a ibas que expresa simultaneidad en el pasado y a habias ido que expresa anterioridad.

La combinación de aspecto y taxis no es tan común tipológicamente, aunque se encuentran algunos ejemplos. Comrie (1976: 78-81) describe un sistema combinado de taxis (en su terminología tiempo relativo) y aspecto en el árabe que es de sumo interés a la luz de los datos de las lenguas mayas. El tradicionalmente llamado "imperfectivo" en el árabe en general se interpreta con referencia al presente o futuro (y los rasgos aspectuales del evento no importan), mientras que el tradicionalmente llamado "perfectivo" normalmente tiene referencia estricta al pasado. No obstante, al igual que en las lenguas mayas, las cláusulas subordinadas de condición o de tiempo permiten el empleo del completivo aun cuando se refieren al futuro. Y cuando por el contexto es evidente que se trata del pasado, el "imperfectivo" puede llevar significado aspectual indicando la 
duración o habitualidad del evento. Así pues, la estructura y semántica del sistema verbal en el árabe efectivamente es muy parecida a lo que se presenta en las lenguas mayas con sistemas verbales binarios que involucran la dicotomía completivo/incompletivo. El árabe puede servir como buena evidencia de que sí existen sistemas verbales cuyos miembros reúnen significados de los dominios semánticos de aspecto y taxis.

\section{UN BOSQUEJO COMPARATIVO}

Como lo hemos visto en $\$ 2$, los rasgos morfológicos de tiempo-aspecto varían mucho enre las lenguas de la familia. Lo mismo sucede con la estructura general del sistema verbal. Dada la variación morfológica y morfosintáctica, uno puede dudar también de la semántica de las categorías de tiempo-aspecto, en tanto que es poco probable que sea la misma en diferentes lenguas mayas.

En $\$ 4.2$ se describió la posibilidad de analizar la semántica del completivo/incompletivo como combinación de significados de taxis y aspecto. Este análisis es conveniente para las lenguas con oposición binaria entre el completivo e incompletivo, como en chontal o tzeltal, por ejemplo, y posiblemente pueda aplicarse al huasteco, chol o k'iché, lenguas en donde el sistema de tiempo-aspecto no se limita al completivo e incompletivo, pero en las que los demás miembros del paradigma -perfecto, progresivo y optativo, respectivamente- con sus significados muy particulares no intervienen en el ámbito semántico del completivo/incompletivo. Pero, por ejemplo, en las lenguas con la categoría del "futuro" o "potencial" la situación ya es diferente porque el significado de taxis 
posterior ya no pertenece al ámbito del incompletivo, y posiblemente no se interpreta del todo como un significado de taxis, sino como tiempo futuro o modo potencial. Esto concierne también a las lenguas con paradigmas de tiempo-aspecto más amplios. Stewart (1979: 187), al describir el sistema verbal en q'eqchí, hace una observación muy importante que en cierta medida puede ser aplicada para otras lenguas mayas: "Un 'afijo de tiempo/aspecto’ puede expresar modo y también puede ser más 'temporal' que 'aspectual', o al revés".

Así pues, para entender la semántica del completivo/incompletivo no es suficiente suponer que indican una combinación de aspecto y taxis; según las lenguas particulares es muy probable que a veces haya que considerar también los ámbitos de modalidad, irrealidad, o distancia temporal. Además, es interesante notar que a veces al analizar una categoría puede ser útil proponer la ausencia de uno u otro significado -véase el análisis del completivo en el maya yucateco propuesto por Bohnemeyer (1998: 327) que dice que el completivo (en su terminología: perfectivo), a diferencia de otras categorías, no lleva ningún significado modal y por lo tanto no se usa en relación al tiempo futuro.

En Vinogradov (2014b: 72) se hace notar que "la semántica del incompletivo varía considerablemente según la lengua particular dentro de la familia maya", y para definirla es necesario tomar en cuenta la semántica de otras marcas que, juntas, constituyen el paradigma de tiempo-aspecto. Lo mismo es correcto para el completivo, aunque la variación semántica del completivo no es tan esencial porque no depende tanto de otras categorías con significados temporales y/o aspectuales que existen en la lengua. Según el estudio de Vinogradov (2014b), se desta- 
can los siguientes tipos semánticos generales del incompletivo dentro de la familia (Tabla 1).

Tabla 1: Tipos semánticos del incompletivo en las lenguas mayas

\begin{tabular}{lll}
\hline & CONSTITUYENTES SEMÁNTICOS & EJEMPLOS DE LENGUAS \\
\hline 1. & Habitual + Progresivo + Futuro/Posterioridad & chontal, tseltal, huasteco \\
2a. & Habitual + Progresivo & '’eqchí', chuj, mam \\
2b. & Habitual + Futuro/Posterioridad & chol \\
3. & Habitual & yucateco, mopán, itzá' \\
\hline
\end{tabular}

A continuación estos tipos se ilustran con ejemplos de tres lenguas: chontal, q'eqchí' y maya yucateco. El chontal presenta un incompletivo semánticamente muy amplio que incluye el aspecto habitual (22a), el aspecto progresivo $(16,22 \mathrm{~b})$ y el significado de posterioridad $(5,22 \mathrm{c})$ que pertenece al dominio de taxis.

(22) Chontal

a. lo ke u=pojl-en=ba mäx ts'ita' DEM REL $3 \mathrm{~A}=$ conseguir-INC=ENCL muy poco 'Lo que conseguía era muy poquito' (Keller \& Luciano 2001: 60, ej. 3)

b. u=chän-i-job u=chikt-an um=p'e noj k'ak' 3A-ver-COM-PL 3A-reflejar-INC uno=CLF grande fuego 'Vieron que alumbraba un gran fuego' (Keller \& Luciano 2001: 69, ej. 132) 
c. ka’jini kä=kol-an

kä=juntuma=la täkä así IPL.A=quedarse-INC IPL.POSS $=$ Solo $=$ INCL también 'Así nos quedaremos solos también' (Keller \& Luciano 2001: 61, ej. 13)

El chontal marca el incompletivo por medio de flexión sufijal (\$2). Hay varios alomorfos del incompletivo -en (22) solo se presentan -en y -an-y su distribución se determina por la clase léxico-sintáctica del verbo; es decir, por la transitividad, agentividad, etc. Para una descripción detallada, consúltese Osorio May (2005).

El q'eqchî dispone de una marca especial para indicar la posterioridad (futuro o potencialidad), y por lo tanto el incompletivo se limita a los significados de habitual (23a) y progresivo (23b), aparentemente combinándolos con el significado de simultaneidad. ${ }^{5}$

Q'EQCHÍ'
a. rajlal kutan na-qa-tiw
keenq' r-ik'in
ik' cada día INC-1PL.ERG-comer frijol 3SG.POSs-con chile 'Cada día comemos frijol con chile'
b. eb' li kok' xul nak-e'-b'ichank ut PL DEF pequeño animal INC-3PL.ABS-cantar y

5 Los ejemplos del q'eqchí provienen del trabajo de campo con hablantes nativos realizado en diciembre del 2014. Expreso mi gratitud a la Fundación de Investigaciones Lingüísticas Fundamentales por haber apoyado mi proyecto de investigación. Cordialmente agradezco a Guillermo Saquil y Alejandro Quib por haberme compartido la riqueza del idioma q’eqchí'. 


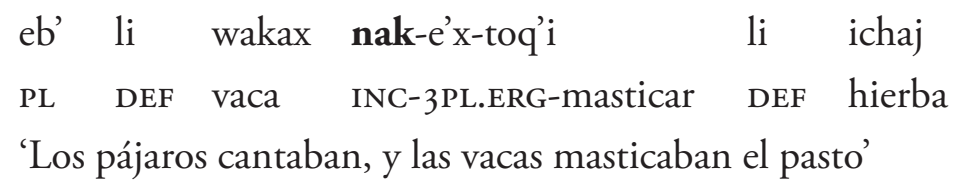

El incompletivo en q'eqchí' tiene tres alomorfos: n-, na-y nak-, y su distribución depende de la marca personal que los sigue. En (23a) antes del prefijo ergativo de la primera persona plural qa-se utiliza el prefijo na-, mientras que en (23b) en ambos casos se presenta la forma completa nak-.

Finalmente, el incompletivo estrecho que no se extiende fuera de la combinación de los significados de habitual y simultaneidad se encuentra en las lenguas mayas de la rama yucatecana (24).

\section{(24) Yucateco \\ sáansamal $\quad \mathbf{k}=\mathrm{u} \quad$ ts’on-ik kéej \\ diariamente $I N C=3 \mathrm{~A}$ cazar-INC venado \\ 'Todos los días cazaba venados' (Andrade \& Máas Collí 1999: 51)}

En (24) se nota la doble marcación del incompletivo en el yucateco conforme a lo que fue dicho en $\$ 2$. El incompletivo se indica por el elemento prepositivo auxiliar $k$ que lleva la marca de persona enclitizada y al mismo tiempo por el sufijo verbal $-i k$.

Así pues, parece que casi siempre se puede interpretar el incompletivo maya en términos de aspecto y taxis, solo que en diferentes lenguas se expresan diferentes significados de estos dominios semánticos y operan diferentes reglas, de preferencia de un dominio al otro en contextos 
particulares. No obstante, otras categorías de tiempo-aspecto pueden requerir el empleo de otros términos para su análisis semántico. En ello se manifiesta la tendencia más o menos general en las lenguas mayas de combinar varios significados de tiempo, aspecto, taxis, distancia temporal y modo, no solo en el marco del paradigma verbal en general, sino también en el marco de las categorías particulares que constituyen este paradigma.

\section{Conclusiones}

Por el momento está claro que el completivo/incompletivo no indican solo tiempo y tampoco solo aspecto. La igualación de la oposición completivo/incompletivo con la del perfectivo/imperfectivo -dos categorías aspectuales registradas en varias lenguas del mundo- únicamente puede complicar más el problema semántico ya que los significados que se expresan por el completivo/incompletivo evidentemente no son los mismos que se sobreentienden tipológicamente cuando se trata de la dicotomía perfectivo/imperfectivo.

En este artículo fueron analizadas varias propuestas para la descripción de la semántica del completivo/incompletivo: como un conjunto de tiempo y aspecto, como categorías modales, en términos de la oposición realis/irrealis y en términos de taxis o tiempo relativo. No se logró optar por alguna de estas alternativas que sirviera indiscutiblemente para todas las lenguas de la familia, aunque la combinación de aspecto y taxis parece ser la hipótesis más probable. Zeisler (2004: 47) en su libro sobre los sistemas de tiempo-aspecto en las lenguas de Tíbet menciona que "muy 
a menudo no le hacen caso al papel que juega la taxis en otras lenguas [no indo-europeas]", y probablemente, esto también puede ser el caso de las lenguas mayas.

Como se mostró en $\$ 5$, diferentes lenguas mayas tienen diferentes sistemas de tiempo-aspecto, tanto en lo que concierne a la morfología y morfosintaxis como al juego de categorías. No es sorprendente que el completivo/incompletivo también difiera semánticamente según la lengua particular. Por lo tanto, se necesita analizar la semántica del completivo/incompletivo por separado en cada lengua de la familia, y un análisis comparativo como el que se da en el presente trabajo por el momento solo puede servir como punto de partida para investigaciones más profundas.

Otro punto relevante para la presente discusión es el desarrollo diacrónico de la semántica del completivo/incompletivo. Si aún aceptamos la propuesta de que el completivo e incompletivo representan los dominios de aspecto y taxis al mismo tiempo, hay que definir los contextos en los que la taxis es más significativa que el aspecto y los contextos en los que el aspecto es más significativo que la taxis. En el árabe -una lengua con sistema verbal muy parecido a los sistemas mayas en lo que concierne a la combinación de aspecto y taxis (véase \$4.2) - según Comrie (1976: 80), "el grado en el que el aspecto o taxis es predominante parece haber cambiado en el transcurso del desarrollo". Hasta el momento desde el punto de vista diacrónico, no se puede postular ninguna hipótesis acerca del caso de las lenguas mayas, ya que no hay estudios semánticos del completivo/incompletivo ni de otras categorías verbales. Ello podría servir como un tema interesante para trabajos futuros. 
Abreviaturas

I, 2, 3 - primera, segunda y tercera persona; A - juego "A" de marcas pronominales; ABS - absolutivo; APPL - aplicativo; ASEG - asegurativo; в - juego "B" de marcas pronominales; $\mathrm{C}$ - juego " $\mathrm{C}$ ” de marcas pronominales; CLF - clasificador; COM - completivo; CONJ - conjunción; DEF - artículo definido; DEM - demostrativo; DIR - direccional; ENCL enclítico; ERG - ergativo; EXIST - existencial; INC - incompletivo; INCL - inclusivo; PASS - pasivo; PL - plural; POSS - posesivo; POT - potencial; PREP - preposición; PROG - progresivo; QUOT - citativo; REFL - reflexivo; REL - relativizador; SG - singular; SUF - sufijo; TR - sufijo transitivizador.

\section{REFERENCIAS}

Alarcón Estrada, Verónica \& Esponda Jimeno, Víctor \& Gómez Gómez, Antonio \& Pérez López, Enrique (eds.). 1997. Cuentos y relatos indigenas, vol. VI. México D.F.: Universidad Nacional Autónoma de México.

Andrade, Manuel \& Máas Collí, Hilaria (eds.). 1999. Cuentos mayas yucatecos, vol. 1. Mérida: Universidad Autónoma de Yucatán.

Ayres, Glenn \& Colby, Benjamin \& Colby, Lore \& Ko’v, Xhas. 2001. El hombre que fue al inframundo porque se preocupó demasiado por la pobreza: Texto Ixil de Nebaj. Tlalocan XIII. 267-312.

Barrett, Edward Rush. 1999. A grammar of Sipakapense Maya. Austin: University of Texas. (Tesis doctoral.) 
Bohnemeyer, Jürgen. 1998. Time relations in discourse: Evidence from a comparative approach to Yukatek Maya. Tilburg: Katholieke Universiteit Brabant. (Tesis doctoral.)

Briceño Chel, Fidencio. 2006. Los verbos del maya yucateco actual: Investigación, clasificación y sistemas conjugacionales. México D.F.: Instituto Nacional de Lenguas Indígenas.

Buenrostro Díaz, Elsa Cristina. 2013a. La voz en Chuj de San Mateo Ixtatán. México D.F.: El Colegio de México. (Tesis doctoral.)

Buenrostro Díaz, Elsa Cristina. 2013b. El éxodo: Una narración en chuj. Tlalocan XIX. 17-58.

Bybee, Joan \& Perkins, Revere \& Pagliuca, William. 1994. The evolution of grammar: Tense, aspect, and modality in the languages of the world. Chicago: The University of Chicago Press.

Comrie, Bernard. 1976. Aspect: An introduction to the study of verbal aspect and related problems. Cambridge: Cambridge University Press.

Comrie, Bernard. 1985. Tense. Cambridge: Cambridge University Press.

Craig, Colette Grinevald. 1977. The structure of Jacaltec. Austin: University of Texas Press.

Dahl, Östen. 1985. Tense and aspect systems. Oxford: Basil Blackwell.

Dayley, Jon P. 1985. Tzutujil grammar. Berkeley: University of California Press.

DeChicchis, Joseph. 1996. Aspect in Q'eqchi' Mayan. Folia Linguistica XXX. 30-59.

Elliott, Jennifer. 2000. Realis and irrealis: Forms and concepts of the grammaticalisation of reality. Linguistic Typology 4(1). 55-90. 
England, Nora C. 1983. A grammar of Mam, a Mayan language. Austin: University of Texas Press.

England, Nora C. 2009. To tell a tale: The structure of narrated stories in Mam, a Mayan language. International Journal of American Linguistics 75(2). 207-231.

England, Nora C. \& Ortiz Domingo, Juan. 1985. Tij T’iiw. Nora C. England collection. The archive of the indigenous languages of Latin America: www.ailla.utexas.org.

Givón, Talmy. 1994. Irrealis and the subjunctive. Studies in Language 18(2). 265-337.

Gvozdanović, Jadranka. 2011. Perfective and imperfective aspect. En Binnick, Robert I. (ed.), The handbook of tense and aspect, 781-802. Oxford: Oxford University Press.

Hofling, Charles Andrew. 2006. A sketch of the history of the verbal complex in Yukatekan Mayan languages. International Journal of American Linguistics 72(3). 367-396.

Houston, Stephen. 1997. The shifting now: Aspect, deixis, and narrative in Classic Maya texts. American Anthropologist 99(2). 291-305. Houston, Stephen \& Robertson, John \& Stuart, David. 2000. The language of Classic Maya inscriptions. Current Anthropology 41(3). 321-356. Jakobson, Roman. 1957. Shifters, verbal categories and the Russian verb. Harvard University: Russian Language Project.

Keller, Kathryn \& Luciano Gerónimo, Plácido. 2001. Textos chontales. Tlalocan XIII. 59-118.

Kettunen, Harri \& Helmke, Cristophe. 2005. Introduction to Maya Hieroglyphs. (Workshop handbook presentado en el $10^{\circ}$ Congreso de 
la Asociación Europea de Mayistas, Leiden, 5-10 de diciembre de 2005.) http://www.mesoweb.com/resources/handbook/WH2005. pdf. (Consultado el 14-04-2015.)

Knowles-Berry, Susan M. \& Hernández Fería, José. 1989. The rabbit and the alligator: A Chontal Mayan text. Tlalocan XI. 227-235.

Kondic, Ana. 2012. Narraciones en huasteco de San Francisco. Tlalocan XVIII. 35-78.

Kuteva, Tania. 1998. On identifying an evasive gram: Action narrowly averted. Studies in Language 22(1). 113-160.

Lacadena, Alfonso \& Davletshin, Albert. 2013. Grammar of Hieroglyphic Maya. (Advanced Workshop Handbook presentado en el Congreso de la Asociación Europea de Mayistas, Brussels, 29-31 de octubre de 2013.)

Laughlin, Robert M. 1977. Of cabbages and kings: Tales from Zinacantán. Washington D.C.: Smithsonian Institution Press.

Law, Danny. 2014. Inferring Aspect in Classic Mayan. (Ponencia presentada en el III Congreso "Form and analysis in Mayan linguistics", México D.F., 4-5 de diciembre de 2014.)

Lehmann, Christian. 1990. Yukatekisch. Zeitschrift für Sprachwissenschaft 9(1-2). 28-51.

Martínez Vázquez, Rafael. 1995. Tiempo relativo en griego antiguo. Philologia Hispalensis 10. 175-197.

Mithun, Marianne. 1995. On the relativity of irreality. En Bybee, Joan L. \& Fleischman, Suzanne (eds.), Modality in grammar and discourse, 367-388. Ámsterdam: John Benjamins. 
Müller, Neele Janna. 2013. Tense, aspect, modality, and evidentiality marking in South American indigenous languages. Utrecht: LOT.

Osorio May, José del Carmen. 2005. Análisis de la morfología verbal del yokotan, "chontal" del poblado de Tecoluta, Nacajuca, Tabasco. México D.F.: Centro de Investigaciones y Estudios Superiores en Antropología Social. (Tesis de maestría.)

Palmer, Frank R. 2001 [1986]. Mood and modality. Cambridge: Cambridge University Press.

Pérez López, Enrique \& Hidalgo Pérez, Manuel \& Gómez Gómez, Antonio (eds.). 1994. Cuentos y relatos indígenas, vol. V. México D.F.: Universidad Nacional Autónoma de México.

Pérez Martínez, Vitalino. 1996. Leyenda Maya Chorti'. Guatemala: Proyecto Lingüístico Francisco Marroquín.

Petrich, Perla (ed.). 1998. Nuestro maíz del Lago Atitlán. Guatemala: Casa de Estudios de los Pueblos del Lago Atitlán.

Polian, Gilles. 2013. Gramática del tseltal de Oxchuc. México D.F.: Centro de Investigaciones y Estudios Superiores en Antropología Social. Plungian, Vladimir A. (Плунгян, Владимир А.) 2004. Predislovie (Предисловие) [Introducción]. En Lander, Yury А. (Ландер, Юрий A.) \& Plungian, Vladimir A. (Плунгян, Владимир A.) \& Urmanchieva, Anna Yu. (Урманчиева, Анна Ю.) (eds.), Irrealis i irreal'nost': Issledovanija po teorii grammatiki (Ирреалис и ирреальность: Исследования по теории грамматики) [Irrealis y irrealidad: Estudios en la teoría de gramática], 9-25. Moscú: Gnosis.

Relatos choles / Albilbä tyi lakty’añ. 2002. México D.F.: Dirección General de Culturas Populares e Indígenas. 
Relatos tzeltales / Lơil a’yej ta Tzeltal kóp. 2002. México D.F.: Dirección General de Culturas Populares e Indígenas.

Roberts, John R. 1990. Modality in Amele and other Papuan languages. Journal of Linguistics 26. 363-401.

Robertson, John. 1992. The history of tenselaspect/mood/voice in the Mayan verbal complex. Austin: University of Texas Press.

Schuller, Rudolf. 1925. La lengua Ts'ots'il: Dialecto del Maya-K'ice,

Chiapas. International Journal of American Linguistics 3(2). 193-218. Stewart, Stephen. 1979. Tense/aspect in Kekchi. En Martin, Laura (ed.), Mayan Linguistics, vol. 3, 185-201. Columbia: University of Missouri. Stoll, Otto. 1888. Die Maya-Sprachen der Pokom-Gruppe, Vol. 1: Die Sprache der Pokonchi-Indianer. Wien: Alfred Hölder.

Ulrich, Matthew \& Ulrich, Rosemary. 1986 [1978]. Mopan Mayan verbs.

Guatemala: Summer Institute of Linguistics.

Vázquez Alvarez, Juan Jesús. 2011. A grammar of Chol, a Mayan language. Austin: University of Texas. (Tesis doctoral.)

Vinogradov, Igor. 2014a. Aspect switching in Tzotzil (Mayan) narratives.

Oklahoma Working Papers in Indigenous Languages 1. 39-54.

Vinogradov, Igor. 2014b. La categoría de incompletivo en las lenguas mayas: Un estudio comparativo de variación semántica. Studii de gramatică contrastivă $21.65-82$.

Watatani, Jun. 1995. Tenselaspect system of Wastek (Mayan). Eugene: University of Oregon. (Tesis de maestría.)

Welmers, William Everett. 1974. African language structures. Berkeley: University of California Press. 
Whittaker, Arabelle \& Warkentin, Viola. 1965. Chol texts on the supernatural. Oklahoma: Summer Institute of Linguistics.

Zavala Maldonado, Roberto. 1992. El Kanjobal de San Miguel Acatán. México D.F.: Universidad Nacional Autónoma de México.

Zeisler, Bettina. 2004. Relative tense and aspectual values in Tibetan languages: A comparative study. Berlin: Walter de Gruyter.

\section{Agradecimientos}

Este artículo se basa parcialmente en las pláticas impartidas en el $17^{\circ}$ Encuentro de Mesoamerikanistas en Basilea, Suiza (31 de enero - 02 de febrero de 2014) y en el $13^{\circ}$ Encuentro Internacional de Lingüística en el Noroeste de México en Hermosillo, Sonora (12-15 de noviembre de 2014). Agradezco a los participantes por sus valiosos comentarios, y a las sugerencias de dos dictaminadores anónimos que ayudaron a mejorar el artículo. Igualmente, expreso mi gratitud al Programa de Becas Posdoctorales en la UNAM. 